\title{
17q21 locus and ORMDL3: an increased risk for childhood asthma
}

\author{
Jennie G. Ono', Tilla S. Worgall ${ }^{2-4}$ and Stefan Worgall ${ }^{1,5}$
}

Genetic variations in the 17q21 locus are strongly associated with childhood nonallergic asthma. Expression of the 17q21 genes, orosomucoid like 3 (ORMDL3) and gasdermin $B(G S M D B)$, is affected by these disease-associated variants. However, until recently, no functional connection of the protein products coded by these genes with asthma was known. Lately, it has been identified that ORMDL3 function has been related to various cellular processes that could be relevant for the pathogenesis of asthma. This includes dysregulation of the unfolded protein response (UPR) associated with airway remodeling and also an effect of ORMDL3-dysregulated sphingolipid synthesis on bronchial hyperreactivity. These findings are crucial for a better understanding of the mechanism of childhood asthma and may lead to asthma therapeutics that target pathways previously not thought to be related to this common pediatric respiratory disease. Furthermore, this may validate the unbiased genome-wide association study (GWAS) approach for complex diseases such as asthma, to better define pathomechanisms and drug targets.

hildhood asthma is a complex genetic disorder (1) that is clinically heterogeneous (2) and poses huge costs to society (3). About half of patients with mild-to-moderate asthma have nonallergic disease (4) and responds poorly to currently available anti-inflammatory therapies (5). Genome-wide association studies (GWAS) have repeatedly and convincingly linked the asthma susceptibility locus $17 q 21$ to nonallergic childhood-onset asthma. The pioneering study by Moffatt et al. (6) characterized more than 317,000 single-nucleotide polymorphisms (SNPs) from 994 patients with a history of childhoodonset asthma and 1,243 nonasthmatics using European family and case-referent panels. This study showed multiple markers at the 17q21 locus to have a strong association with childhoodonset asthma (combined $P$ value $<10^{-12}$ ), which was then replicated in German and British cohorts. The strongest association was mapped to a $206 \mathrm{~kb}$ interval on chromosome $17 \mathrm{q} 21$; the SNP with the strongest association within this interval was rs72163891 and has been widely reproducible. The association of SNPs in this region with asthma was stronger for early-onset asthma as shown in all but one study (7).

\section{GENE EXPRESSION}

Because polymorphisms in regulating elements could alter gene transcription of factors related to the susceptibility for asthma, the expression of genes coded for in the asthmaassociated region on $17 \mathrm{q} 21$ was evaluated. Moffatt et al. (6) showed that in Epstein-Barr virus-transformed lymphoblastoid cell lines, transcript levels of the orosomucoid like 3 gene (ORMDL3) were strongly and positively associated to rs7216389, the SNP with the strongest association with childhood asthma. This suggested that variants at this asthma susceptibility locus may regulate ORMDL3 expression, which has also been confirmed in rhinovirus-infected blood cells (8). The 17 q21 asthma susceptibility locus is located between 35.0 and $35.5 \mathrm{Mb}$ on chromosome 17 and contains at least 15 genes. To date, however, asthma-associated SNPs have been associated with the expression of only four of these genes (9): (i) Ikaros zinc finger protein 3 (IKZF3), involved with the regulation of lymphocyte development; (ii) Gasdermin B (GSDMB), implicated in epithelial cell barrier function; (iii) Mediator of RNA polymerase II transcription subunit 24 (MED24), a component of a transcriptional coactivator complex thought to be required for expression of most genes; and (4) ORMDL3, an endoplasmic reticulum (ER) transmembrane protein involved in regulation of sphingolipid metabolism. GSDMB and ORMDL3 have received the most attention and their genotype-mediated expression is also affected by rhinovirus infection, one of the most common and powerful triggers for asthma exacerbations (8). It has further been suggested that these two genes might be coregulated, as their transcript levels seem connected (10).

\section{ASSOCIATION OF THE 17q21 LOCUS WITH ASTHMA TYPES}

The GABRIEL consortium, a multidisciplinary study to identify genetic and environmental causes of asthma in the European Community, followed up the initial identification of the $17 \mathrm{q} 21$ locus with a meta-analysis of GWAS from 10,365 subjects with asthma and 16,110 unaffected subjects, all of European descent. SNPs within the ORMDL3/GSDMB asthma-susceptibility locus achieved genome-wide level significance specifically with childhood-onset asthma but not adult-onset asthma (11). Since then, this region has been consistently replicated 
as an asthma susceptibility locus in ethnically distinct populations, including Mexicans, Puerto Ricans and AfricanAmericans (12), Japanese (13), Chinese (14,15), Scottish (16), European (16-18), and French Canadians (19). In the United States, the EVE consortium confirmed the 17q21 locus in nine GWAS datasets, representing three major ethnic groups (European American, African American or African Carribean, and Latino ancestry), although no single SNP was associated with asthma in all three ethnic groups (20).

The phenotypic relationship of asthma with atopy and allergic sensitization is well established, but the exact nature of this relationship still remains unclear. The overlap between allergy, atopy, and asthma is highest in childhood asthma, with estimates for up to $80 \%$ of asthmatic children being allergic, but seems lower in adults (60\%) (21). It remains unknown, however, if asthma and atopy are a result of the same disease process, or if they represent distinct disease entities occurring concomitantly with different causes (1). Analyses by the GABRIEL consortium revealed no association between the ORMDL3/GSDMB locus and IgE levels, a commonly used indicator for atopy (11). This has been replicated in numerous studies $(8,9,13,18,22,23)$. The absence of any overlap between the most highly associated asthma-susceptible SNPs and serum IgE at any locus tested suggests separate, genetically distinct pathways. Instead, $17 \mathrm{q} 21$ SNPs have been correlated with bronchial hyperreactivity (9), a cardinal feature of asthma. This suggests that the disease susceptibility originating from the $17 \mathrm{q} 21$ locus affects airway reactivity independent of allergic sensitization.

\section{LINKING GENE(S) TO FUNCTION(S)}

None of the genes in the asthma-associated $17 \mathrm{q} 21$ region would have been associated to asthma without the unbiased GWAS approach. This approach is an opportunity to discover novel targets and pathomechanisms but has often been both a challenge and a curse for complex diseases. This is particularly true for ORMDL3. Until recently, little was known about its overall cellular function in mammalian cells, as most of its function had been studied in yeast. There, ORMDL functions as regulator of sphingolipid synthesis (24). Since the asthmaassociated SNPs lead to increased cellular ORMDL3 protein expression, it would suggest that an asthma phenotype related to ORMDL3 should be associated with a gain-of-protein function. To date, the following mechanisms of how increased ORMDL3 could be related to asthma have been proposed (Figure 1): (i) ORMDL3 is involved in ER-mediated $\mathrm{Ca}^{2+}$ signaling and activation of the unfolded protein response (UPR), leading to epithelial cell remodeling through its effect on the sarco/endoplasmic reticulum CaATPase (SERCA) $(25,26)$ and (ii) ORDML3 influences sphingolipid metabolism to directly affect bronchial reactivity (27).

\section{ORMDL3 AND INTRACELLULAR CALCIUM HOMEOSTASIS}

ORMDL3 is widely expressed in both fetal and adult mammalian tissues including lung epithelial cells $(26,28)$. In mouse lungs, expression can be increased by a variety of stimuli, such as allergens, tobacco smoke, and lipopolysaccharides (26). In lung

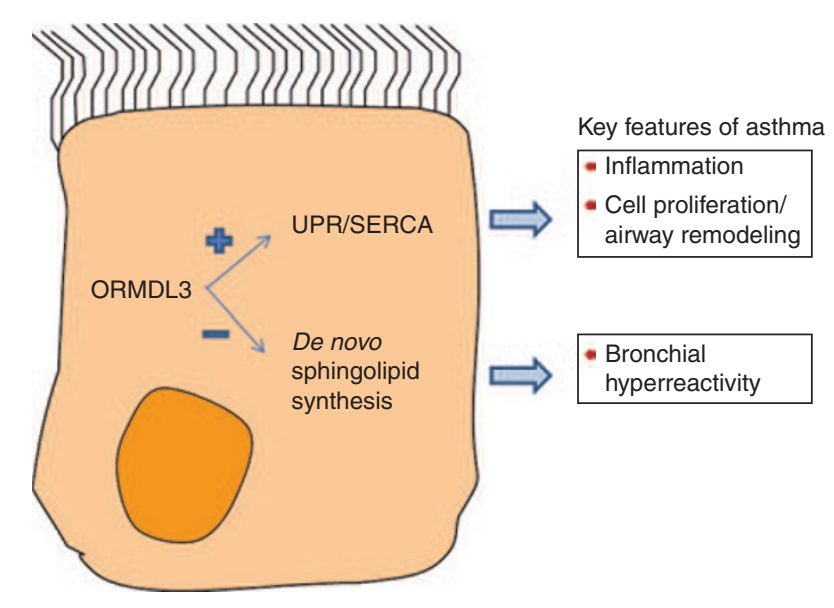

Figure 1. Proposed mechanisms for the role of ORMDL3 in asthma pathogenesis in an airway epithelial cell. ORMDL3, orosomucoid like 3; SERCA, sarco/endoplasmic reticulum Ca ATPase; UPR, unfolded protein response.

epithelial cells, in vitro expression can be increased by interleukin-4 (IL-4) and interleukin-13 (IL-13) but not tumor necrosis factor- $\alpha$ (26). Interestingly, overexpression of ORMDL3 in airway epithelial cells activates activating transcription factor 6 (ATF6), one of the three signaling branches of the UPR in response to ER stress. This appears to be accompanied by increases in metalloproteases (MMP-9, ADAM-8), CC chemokines (CCL-20), CXC chemokines (IL-8, CXCL-10, CXCL-11), and oligoadenylate synthetase (OAS). These findings suggest that ORMDL3 is an allergen- and cytokine-inducible gene that may regulate the expression of chemokines, metalloproteinases, and OAS through activation of the UPR and may thus be linked to inflammatory and remodeling responses in asthma (26). Activation of the UPR has been implicated in other inflammatory and immune-related diseases other than asthma, such as inflammatory bowel disease, chronic obstructive pulmonary disease, and diabetes, as well as environmental stressors such as tobacco smoke, an important trigger for asthma exacerbations. It is unclear, if activation of the UPR is a primary underlying mechanism of asthma or a reaction to chronic inflammation or environmental insults (29).

Proper assembly and folding of ER proteins is dependent on appropriate $\mathrm{ER} \mathrm{Ca}^{2+}$ levels, with alterations of these resulting in activation of the UPR. The UPR is a system of highly conserved signaling pathways, which sense the needs and capacity of the ER to maintain protein quality control and homeostasis. The ER responds to unfolded proteins in its lumen by activating intracellular signal transduction pathways which allow for expansion of the ER protein folding machinery. If these needs are not met, apoptosis is induced (29). The ER stress response is also involved in inflammation through the activation of transcriptional regulators of genes involved in the inflammatory response (30). Of the three primary UPR transcription pathways such as, ATF6, double-stranded RNA-activated protein kinase (PKR)-like endoplasmic reticulum kinase (PERK), and inositol requiring kinase 1 (IRE1), ATF6 has been shown to regulate SERCA (26) and interleukin-6 (IL-6) (31), both of which have been implicated in the pathogenesis of asthma. 


\section{\begin{tabular}{l|l} 
ORMDL3 expression and childhood asthma & Review
\end{tabular}}

SERCA belong to the P-type ATPase family to actively transport cations across membranes and play a key role in $\mathrm{Ca}^{2+}$ signaling by restoring free $\mathrm{Ca}^{2+}$ to baseline levels after cell activation and by replenishing sarcoplasmic reticulum stores (32). The SERCA2b isoform is mainly expressed in smooth muscle cells and has been shown to be the predominate isoform in airway smooth muscle cells (25). Smooth muscle responses to a variety of stimuli are controlled by changes in the concentration of free cytosolic $\mathrm{Ca}^{2+}$. Alterations in the control of smooth muscle cell $\mathrm{Ca}^{2+}$ concentration might be an important contributor to airway hyperresponsiveness in asthma (33). Decreased SERCA2 expression as well as a diminished ability of SERCA2 to replete sarcoplasmic reticulum (SR) $\mathrm{Ca}^{2+}$ stores has been seen in asthmatic airway smooth muscle, suggesting that $\mathrm{Ca}^{2+}$ handling in airway smooth muscle may be abnormal in asthma and that disruption of SERCA2 contributes to the increased proliferation and enhanced cytokine expression in airway smooth muscle (25). However, some of the data relating ORMDL3 to this has been conflicting: Miller et al. (26) showed that overexpression of ORMDL3 selectively activates the ATF6 and that knockdown of ATF6 decreases expression of SERCA. By contrast, Cantero-Recasens et al. (34) showed that overexpression of ORMDL3 impaired cytosolic $\mathrm{Ca}^{2+}$ clearance, resulting in an inhibitory effect on SERCA activity. The group also showed coimmunoprecipitation of ORMDL3 and SERCA and that ORMDL3 activates the UPR through the PERK pathway with no effect on the IRE1 pathway (34). In contrast, Hsu et al. (35) found no association of ORMDL3 expression with UPR activation or with changes in IL-6 and IL-8. In addition, a study in a T-cell line demonstrated that ORMDL3 decreased cell activation by decreasing store-operated $\mathrm{Ca}^{2+}$ entry, an important mechanism of $\mathrm{Ca}^{2+}$ homeostasis and activation in immune cells (36). This would suggest that higher ORMLD3 expression is associated with decreased T-cell activation. Taken together, these studies strongly suggest a relationship between ORMDL3, SERCA, regulation of intracellular $\mathrm{Ca}^{2+}$, and the UPR, which is still unfolding. Of note is that none of these studies address why ORMDL3 polymorphisms would be linked more to nonallergic asthma, nor do they study a potential effect on sphingolipids, the class of cellular lipids that is regulated by ORMDL3.

\section{ORMDL3 AND SPHINGOLIPIDS}

A role of orm proteins in the regulation of sphingolipid synthesis has been well established in yeast for many years $(24,28,37-$ $39)$ and has recently been demonstrated in human cells $(40,41)$. ORMDL proteins act as negative regulators of sphingolipid synthesis by interacting with serine palmitoyl-coenzyme A transferase (SPT) (38). SPT catalyzes the condensation of serine and palmitoyl-coenzyme $\mathrm{A}$, the rate-limiting step of the de novo sphingolipid synthesis (Figure 2). The reaction product, 3-ketosphinganine is instable. It is converted to sphinganine that is metabolized by distinct ceramide synthases to dihydroceramides. A dihydroceramide desaturase generates ceramides that also originate from catabolism of complex sphingolipids (i.e. sphingomyelin, gangliosides) $(42,43)$. Sphingolipids are a diverse and complex category of lipids due to their numerous

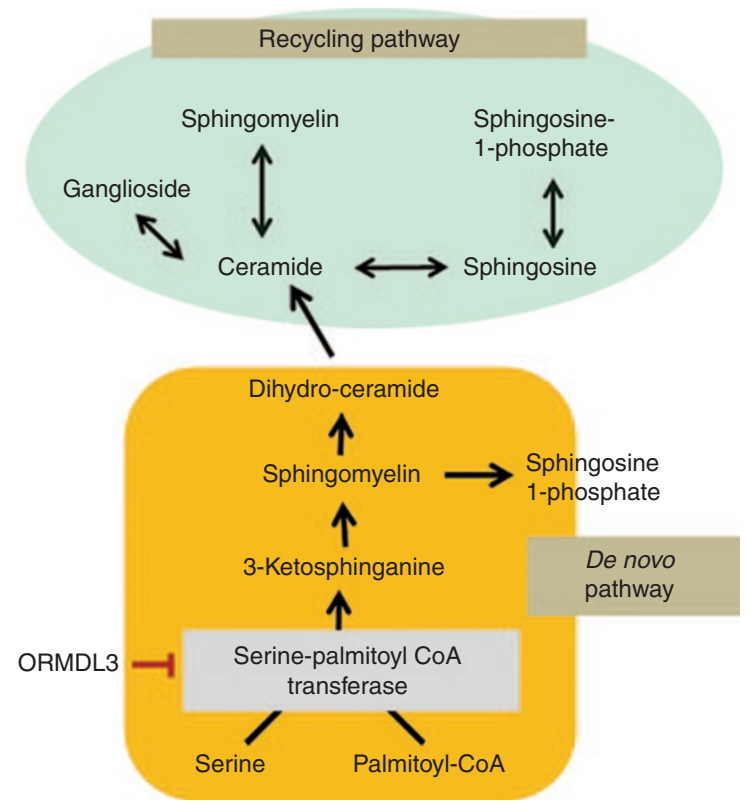

Figure 2. Sphingolipid synthesis. Highlighted are de novo and recycling pathways of sphingolipid metabolism.

variations in the sphingoid bases, fatty acids, and head groups $(37,44,45)$. Several sphingolipids, in particular, ceramides, sphingosine, and sphingosine 1-phosphate (S1P), function as bioactive signaling molecules $(37,44)$. The regulation of sphingolipid metabolism is incompletely understood and likely complex through multiple interconnected mechanisms (37). Although de novo sphingolipid synthesis may only play a minor contribution to the total cellular sphingolipid pool (46), homeostatic regulators of de novo synthesis such as ORMDL stabilize cellular sphingolipid levels in the face of external perturbations $(37,38,47)$. Previous studies linking asthma to sphingolipids have been centered on inflammatory and allergic mechanisms related to the sphingolipid mediator S1P (48-53). S1P is involved in mast cell degranulation and airway hyperresponsiveness in allergic asthma models (53-56) and has been a focus on the development of sphingolipid-based anti-inflammatory agents (51,57-59).

The asthma-associated ORMDL3 SNPs are associated with higher expression of ORMDL. Knock-down of ORMDL1, 2, and 3 in mammalian cells increases ceramides, products of sphingolipid synthesis (38). Therefore, asthma-associated SNPs are expected to negatively regulate SPT resulting in inhibited de novo sphingolipid synthesis (46). A recent study from our laboratories suggest that impaired de novo sphingolipid synthesis leads to airway hyperreactivity in mouse lungs and both human and murine bronchial rings. In addition, we observed altered magnesium homeostasis and contractile response to magnesium (27). SPT activity in the lung was decreased using myriocin, a specific inhibitor of SPT, or using a genetic model of SPT-haploinsufficient mice. The associated increased airway hyperreactivity was not associated with inflammation or mucus hyperplasia. These findings are also supported by the recently reported association of SNPs in the 17q21 locus with 
bronchial hyperreactivity without atopy (9). Airway remodeling in asthma is commonly considered to be a consequence of sustained airway inflammation (60). This assumption has recently been challenged in such that a genetically predisposed asthmatic respiratory tract reacts in a certain way to environmental (allergic or inflammatory) stimuli (61-63). Our study suggests that genetically altered sphingolipid homeostasis could be one of those predispositions. Genetic deficiency of SPT has not been observed in humans. Of note is a recent description of two nonrelated children with a currently undefined metabolic defect and encephalopathy characterized by low serine concentrations (the substrate for SPT) in serum and CSF, both of whom were also suffered from severe asthma (64).

Sphingolipids affect the force of skeletal muscle contraction $(65,66)$ and smooth muscle proliferation $(67)$. The effect of altered cellular sphingolipids on airway smooth muscle cells is not known. The association of decreased de novo sphingolipid synthesis with alterations in cellular magnesium homeostasis and the altered contractile sensitivity to magnesium could provide a mechanistic link of decreased de novo sphingolipid synthesis to smooth muscle function. Asthma has been associated with lower intracellular magnesium concentrations $(68,69)$, and intravenous or nebulized magnesium sulfate $\left(\mathrm{MgSO}_{4}\right)$ is used to treat asthma exacerbations $(68,70)$. This therapeutic approach has been very controversial with variable efficacy. In light of these new findings, it could be speculated that response to $\mathrm{MgSO}_{4}$ depends on the $17 \mathrm{q} 21$ genotype.

\section{ENVIRONMENTAL INFLUENCE AND GENETIC SUSCEPTIBILITY}

The development and clinical course of childhood asthma is impacted by environmental exposures. Environmental exposures linked to asthma risk include tobacco smoke, day care attendance in early childhood, exposure to farms and farm animals, respiratory viral infections, allergens, and others (1). Although the exact mechanisms by which environmental exposures affect asthma remain unclear, epigenetic mechanisms likely play an important role, affecting the regulation of genes accessible for transcription through the modification of DNA and DNA-associated proteins. Early viral infections are well-known, but poorly understood, asthma risk factors, and it appears that variants at the 17q21 locus may enhance the association between early respiratory infections and childhood asthma. In a study by Smit et al. (71), the association between early viral infection and asthma showed a greater than twofold difference in odds ratio in individuals who were homozygous for minor alleles at the ORMDL3-associated SNPs. Respiratory infections with rhinovirus, one of the most potent triggers of asthma exacerbations, but not with respiratory syncytial virus were associated with a $>10$-fold increase in odds ratio for childhood asthma in individuals with the at-risk genotype rs7216389-TT (8). This highlights the interaction between genetic susceptibility and a common environmental risk factor. The increased risk for early-onset asthma conferred by the $17 q 21$ locus was further increased by early exposure to tobacco smoke (18) and also confirmed in a study analyzing the relationship between early-onset asthma, viral infection, and tobacco smoke exposure (71).

Exposure to farm environments seems to have a protective effect on asthma and the development of allergies (72). DNA methylation patterns observed in cord blood, which were not found at $4 \mathrm{y}$ of age in a large rural birth cohort, showed hypermethylation in loci related to ORMDL3, and also signal transducer and activator of transcription 6 (STAT6), in asthmatics from nonfarm environments as compared with nonasthmatic farm children (74). This suggests that epigenetic influences present at birth, but not after $4 \mathrm{y}$ of age, may influence disease susceptibility. Interestingly, the asthmatic farmers' children had a significant decrease in methylation of the ORMDL3 loci over time as compared with increasing methylation in asthmatic children of nonfarmers, suggesting an environmental effect on epigenetic changes in the 17q21 locus (74).

\section{CONCLUSIONS}

None of the current medications for asthma are disease modifying or curative (73). Despite tremendous efforts to find an underlying cause for asthma that can be targeted therapeutically, none of these have yet been successful. GWAS studies using the most unbiased approach to find the genetic origin of asthma have also not yet been successful. Remarkably though is the consistency with which the 17q21 locus and within it ORMDL3 has been associated with asthma. Without this approach, it is unlikely that the genes in this region would have been primarily identified to be associated with asthma. It is becoming increasingly clear that this locus is associated with early-onset asthma and bronchial hyperreactivity, independent of allergic sensitization and wheezing phenotypes. Although the functional connection of altered ORMDL3 expression has not been made in humans, recent experimental data linking this protein's effect on asthma to altered calcium homeostasis, UPR, airway remodeling, and sphingolipid synthesis open new therapeutic targets for asthma at its basic mechanisms. Current asthma therapies rely mainly on bronchodilators for symptom relief and on anti-inflammatory actions of steroids and other anti-inflammatory medications. Identifying novel pathways that could be therapeutically manipulated based on a patient's genotype is an important step toward a more personalized medicine approach to asthma therapies.

\section{STATEMENT OF FINANCIAL SUPPORT}

J.G.O. is supported by a grant UL1 TR000457 from the National Center for Advancing Translational Sciences, National Institutes of Health. No other financial assistance was received to support this study.

\section{REFERENCES}

1. Ober C, Yao TC. The genetics of asthma and allergic disease: a $21^{\text {st }}$ century perspective. Immunol Rev 2011;242:10-30.

2. Gary PA. Endotyping asthma: new insights into key pathogenic mechanisms in a complex, heterogeneous disease. Lancet 2008;372:1107-19.

3. Barnett SB, Nurmagambetov TA. Costs of asthma in the United States: 2002-2007. J Allergy Clin Immunol 2011;127:145-52.

4. McGrath KW, Icitovic N, Boushey HA, et al.; Asthma Clinical Research Network of the National Heart, Lung, and Blood Institute. A large subgroup of mild-to-moderate asthma is persistently noneosinophilic. Am J Respir Crit Care Med 2012;185:612-9. 


\section{ORMDL3 expression and childhood asthma $\quad$ Review}

5. Paul MOB. Therapeutic strategies to reduce asthma exacerbations. J Allergy Clin Immunol 2011;128:257-63.

6. Moffatt MF, Kabesch M, Liang L, et al. Genetic variants regulating ORMDL3 expression contribute to the risk of childhood asthma. Nature 2007;448:470-3.

7. Flory JH, Sleiman PM, Christie JD, et al. 17q12-21 variants interact with smoke exposure as a risk factor for pediatric asthma but are equally associated with early-onset versus late-onset asthma in North Americans of European ancestry. J Allergy Clin Immunol 2009;124:605-7.

8. Caliskan M, Bochkov YA, Kreiner-Møller E, et al. Rhinovirus wheezing illness and genetic risk of childhood-onset asthma. N Engl J Med 2013;368:1398-407.

9. Granell R, Henderson AJ, Timpson N, et al. Examination of the relationship between variation at 17q21 and childhood wheeze phenotypes. J Allergy Clin Immunol 2013;131:685-94.

10. Verlaan DJ, Berlivet S, Hunninghake GM, et al. Allele-specific chromatin remodeling in the ZPBP2/GSDMB/ORMDL3 locus associated with the risk of asthma and autoimmune disease. Am J Hum Genet 2009;85:37793.

11. Moffatt MF, Gut IG, Demenais F, et al.; GABRIEL Consortium. A largescale, consortium-based genomewide association study of asthma. N Engl J Med 2010;363:1211-21.

12. Galanter J, Choudhry S, Eng C, et al. ORMDL3 gene is associated with asthma in three ethnically diverse populations. Am J Respir Crit Care Med 2008; 177:1194-200.

13. Hirota T, Harada M, Sakashita M, et al. Genetic polymorphism regulating ORM1-like 3 (Saccharomyces cerevisiae) expression is associated with childhood atopic asthma in a Japanese population. J Allergy Clin Immunol 2008;121:769-70

14. Leung TF, Sy HY, Ng MC, et al. Asthma and atopy are associated with chromosome 17q21 markers in Chinese children. Allergy 2009;64:621-8.

15. Yang FF, Huang Y, Li QB, Dai JH, Fu Z. Single nucleotide polymorphisms in the ORM1-like 3 gene associated with childhood asthma in a Chinese population. Genet Mol Res 2012;11:4646-53.

16. Tavendale R, Macgregor DF, Mukhopadhyay S, Palmer CN. A polymorphism controlling ORMDL3 expression is associated with asthma that is poorly controlled by current medications. J Allergy Clin Immunol 2008;121:860-3.

17. Sleiman PM, Annaiah K, Imielinski M, et al. ORMDL3 variants associated with asthma susceptibility in North Americans of European ancestry. J Allergy Clin Immunol 2008;122:1225-7.

18. Bouzigon E, Corda E, Aschard $\mathrm{H}$, et al. Effect of $17 \mathrm{q} 21$ variants and smoking exposure in early-onset asthma. N Engl J Med 2008;359:1985-94.

19. Madore AM, Tremblay K, Hudson TJ, Laprise C. Replication of an association between 17q21 SNPs and asthma in a French-Canadian familial collection. Hum Genet 2008;123:93-5.

20. Torgerson DG, Ampleford EJ, Chiu GY, et al.; Mexico City Childhood Asthma Study (MCAAS); Children's Health Study (CHS) and HARBORS study; Genetics of Asthma in Latino Americans (GALA) Study, Study of Genes-Environment and Admixture in Latino Americans (GALA2) and Study of African Americans, Asthma, Genes \& Environments (SAGE); Childhood Asthma Research and Education (CARE) Network; Childhood Asthma Management Program (CAMP); Study of Asthma Phenotypes and Pharmacogenomic Interactions by Race-Ethnicity (SAPPHIRE); Genetic Research on Asthma in African Diaspora (GRAAD) Study. Meta-analysis of genome-wide association studies of asthma in ethnically diverse North American populations. Nat Genet 2011;43:88792.

21. Johansson SG, Lundahl J. Asthma, atopy, and IgE: what is the link? Curr Allergy Asthma Rep 2001;1:89-90.

22. Binia A, Khorasani N, Bhavsar PK, et al. Chromosome 17q21 SNP and severe asthma. J Hum Genet 2011;56:97-8.

23. Bisgaard H, Bønnelykke K, Sleiman PM, et al. Chromosome 17q21 gene variants are associated with asthma and exacerbations but not atopy in early childhood. Am J Respir Crit Care Med 2009;179:179-85.

24. Han S, Lone MA, Schneiter R, Chang A. Orm1 and Orm2 are conserved endoplasmic reticulum membrane proteins regulating lipid homeostasis and protein quality control. Proc Natl Acad Sci USA 2010;107:5851-6.
25. Mahn K, Hirst SJ, Ying S, et al. Diminished sarco/endoplasmic reticulum $\mathrm{Ca} 2+$ ATPase (SERCA) expression contributes to airway remodelling in bronchial asthma. Proc Natl Acad Sci USA 2009;106:10775-80.

26. Miller M, Tam AB, Cho JY, et al. ORMDL3 is an inducible lung epithelial gene regulating metalloproteases, chemokines, OAS, and ATF6. Proc Natl Acad Sci USA 2012;109:16648-53.

27. Worgall TS, Veerappan A, Sung B, et al. Impaired sphingolipid synthesis in the respiratory tract induces airway hyperreactivity Sci Transl Med 2013;186:186ra67.

28. Hjelmqvist L, Tuson M, Marfany G, Herrero E, Balcells S, Gonzàlez-Duarte R. ORMDL proteins are a conserved new family of endoplasmic reticulum membrane proteins. Genome Biol 2002;3:RESEARCH0027.

29. Walter P, Ron D. The unfolded protein response: from stress pathway to homeostatic regulation. Science 2011;334:1081-6.

30. Hotamisligil GS. Endoplasmic reticulum stress and the inflammatory basis of metabolic disease. Cell 2010;140:900-17.

31. Adachi Y, Yamamoto K, Okada T, Yoshida H, Harada A, Mori K. ATF6 is a transcription factor specializing in the regulation of quality control proteins in the endoplasmic reticulum. Cell Struct Funct 2008;33:75-89.

32. Hovnanian A. SERCA pumps and human diseases. Subcell Biochem 2007;45:337-63.

33. Parameswaran K, Janssen LJ, O'Byrne PM. Airway hyperresponsiveness and calcium handling by smooth muscle: a "deeper look". Chest 2002;121:621-4.

34. Cantero-Recasens G, Fandos C, Rubio-Moscardo F, Valverde MA, Vicente $\mathrm{R}$. The asthma-associated ORMDL3 gene product regulates endoplasmic reticulum-mediated calcium signaling and cellular stress. Hum Mol Genet 2010;19:111-21.

35. Hsu KJ, Turvey SE. Functional analysis of the impact of ORMDL3 expression on inflammation and activation of the unfolded protein response in human airway epithelial cells. Allergy Asthma Clin Immunol 2013;9:4.

36. Carreras-Sureda A, Cantero-Recasens G, Rubio-Moscardo F, et al. ORMDL3 modulates store-operated calcium entry and lymphocyte activation. Hum Mol Genet 2013;22:519-30.

37. Breslow DK, Weissman JS. Membranes in balance: mechanisms of sphingolipid homeostasis. Mol Cell 2010;40:267-79.

38. Breslow DK, Collins SR, Bodenmiller B, et al. Orm family proteins mediate sphingolipid homeostasis. Nature 2010;463:1048-53.

39. Roelants FM, Breslow DK, Muir A, Weissman JS, Thorner J. Protein kinase Ypk1 phosphorylates regulatory proteins Orm1 and Orm2 to control sphingolipid homeostasis in Saccharomyces cerevisiae. Proc Natl Acad Sci USA 2011;108:19222-7.

40. Siow DL, Wattenberg BW. Mammalian ORMDL proteins mediate the feedback response in ceramide biosynthesis. J Biol Chem 2012;287:40198-204.

41. Halapi E, Gudbjartsson DF, Jonsdottir GM, et al. A sequence variant on $17 \mathrm{q} 21$ is associated with age at onset and severity of asthma. Eur J Hum Genet 2010;18:902-8.

42. Hanada K. Serine palmitoyltransferase, a key enzyme of sphingolipid metabolism. Biochim Biophys Acta 2003;1632:16-30.

43. Merrill AH Jr. De novo sphingolipid biosynthesis: a necessary, but dangerous, pathway. J Biol Chem 2002;277:25843-6.

44. Hannun YA, Obeid LM. Principles of bioactive lipid signalling: lessons from sphingolipids. Nat Rev Mol Cell Biol 2008;9:139-50.

45. Merrill AH Jr, Sandhoff K. Sphingolipids: metabolism and cell signaling. In: Dennis E, Vance JEV, eds. New Comprehensive Biochemistry, vol. 36. Elsevier, 2002:373-407.

46. Walther TC. Keeping sphingolipid levels nORMal. Proc Natl Acad Sci USA 2010;107:5701-2.

47. Sun Y, Miao Y, Yamane Y, et al. Orm protein phosphoregulation mediates transient sphingolipid biosynthesis response to heat stress via the Pkh-Ypk and Cdc55-PP2A pathways. Mol Biol Cell 2012;23:2388-98.

48. Ammit AJ, Hastie AT, Edsall LC, et al. Sphingosine 1-phosphate modulates human airway smooth muscle cell functions that promote inflammation and airway remodeling in asthma. FASEB J 2001; 15:1212-4.

49. Fyrst H, Saba JD. An update on sphingosine-1-phosphate and other sphingolipid mediators. Nat Chem Biol 2010;6:489-97. 
50. Jolly PS, Rosenfeldt HM, Milstien S, Spiegel S. The roles of sphingosine1-phosphate in asthma. Mol Immunol 2002;38:1239-45.

51. Lai WQ, Goh HH, Bao Z, Wong WS, Melendez AJ, Leung BP. The role of sphingosine kinase in a murine model of allergic asthma. J Immunol 2008;180:4323-9.

52. Rosenfeldt HM, Amrani Y, Watterson KR, Murthy KS, Panettieri RA Jr, Spiegel S. Sphingosine-1-phosphate stimulates contraction of human airway smooth muscle cells. FASEB J 2003;17:1789-99.

53. Roviezzo F, D’Agostino B, Brancaleone V, et al. Systemic administration of sphingosine-1-phosphate increases bronchial hyperresponsiveness in the mouse. Am J Respir Cell Mol Biol 2010;42:572-7.

54. Kume H, Takeda N, Oguma T, et al. Sphingosine 1-phosphate causes airway hyper-reactivity by rho-mediated myosin phosphatase inactivation. J Pharmacol Exp Ther 2007;320:766-73.

55. Roviezzo F, Di Lorenzo A, Bucci M, et al. Sphingosine-1-phosphate/sphingosine kinase pathway is involved in mouse airway hyperresponsiveness. Am J Respir Cell Mol Biol 2007;36:757-62.

56. Ryan JJ, Spiegel S. The role of sphingosine-1-phosphate and its receptors in asthma. Drug News Perspect 2008;21:89-96.

57. Blé FX, Cannet C, Zurbruegg S, et al. Activation of the lung S1P(1) receptor reduces allergen-induced plasma leakage in mice. $\mathrm{Br} \mathrm{J}$ Pharmacol 2009;158:1295-301.

58. Lai WQ, Wong WS, Leung BP. Sphingosine kinase and sphingosine 1-phosphate in asthma. Biosci Rep 2011;31:145-50.

59. Nishiuma T, Nishimura Y, Okada T, et al. Inhalation of sphingosine kinase inhibitor attenuates airway inflammation in asthmatic mouse model. Am J Physiol Lung Cell Mol Physiol 2008;294:L1085-93.

60. Tagaya E, Tamaoki J. Mechanisms of airway remodeling in asthma. Allergol Int 2007;56:331-40.

61. Lambrecht BN, Hammad H. The airway epithelium in asthma. Nat Med 2012;18:684-92.

62. Malmström K, Pelkonen AS, Mäkelä MJ. Remodeling, inflammation and airway responsiveness in early childhood asthma. Curr Opin Allergy Clin Immunol 2013;13:203-10.
63. Tourdot S, Mathie S, Hussell T, et al. Respiratory syncytial virus infection provokes airway remodelling in allergen-exposed mice in absence of prior allergen sensitization. Clin Exp Allergy 2008;38:1016-24.

64. Keularts IM, Leroy PL, Rubio-Gozalbo EM, et al. Fatal cerebral edema associated with serine deficiency in CSF. J Inherit Metab Dis 2010;33:Suppl $3:$ S181-5.

65. Nikolova-Karakashian MN, Reid MB. Sphingolipid metabolism, oxidant signaling, and contractile function of skeletal muscle. Antioxid Redox Signal 2011;15:2501-17.

66. Sabbadini RA, Betto R, Teresi A, Fachechi-Cassano G, Salviati G. The effects of sphingosine on sarcoplasmic reticulum membrane calcium release. J Biol Chem 1992;267:15475-84.

67. Jacobs LS, Kester M. Sphingolipids as mediators of effects of plateletderived growth factor in vascular smooth muscle cells. Am J Physiol 1993;265(3 Pt 1):C740-7.

68. Dominguez LJ, Barbagallo M, Di Lorenzo G, et al. Bronchial reactivity and intracellular magnesium: a possible mechanism for the bronchodilating effects of magnesium in asthma. Clin Sci 1998;95:137-42.

69. Hashimoto Y, Nishimura Y, Maeda H, Yokoyama M. Assessment of magnesium status in patients with bronchial asthma. J Asthma 2000;37:489-96.

70. Kowal A, Panaszek B, Barg W, Obojski A. The use of magnesium in bronchial asthma: a new approach to an old problem. Arch Immunol Ther Exp (Warsz) 2007;55:35-9.

71. Smit LA, Bouzigon E, Pin I, et al.; EGEA Cooperative Group. 17q21 variants modify the association between early respiratory infections and asthma. Eur Respir J 2010;36:57-64.

72. Ege MJ, Mayer M, Normand AC, et al.; GABRIELA Transregio 22 Study Group. Exposure to environmental microorganisms and childhood asthma. N Engl J Med 2011;364:701-9.

73. Barnes PJ. New drugs for asthma. Semin Respir Crit Care Med 2012;33:68594.

74. Michel S, Busato F, Genuneit J, et al.; PASTURE study group. Farm exposure and time trends in early childhood may influence DNA methylation in genes related to asthma and allergy. Allergy 2013;68:355-64. 